\title{
Counting deficits or diseases? The agreement between frailty and multimorbidity in subjects with cognitive disturbances
}

\author{
Marco Canevelli ${ }^{1}$ (1) $\cdot$ Riccardo Raganato $^{1} \cdot$ Francesca Remiddi $^{1} \cdot$ Federica Quarata ${ }^{1} \cdot$ Martina Valletta $^{1}$. \\ Giuseppe Bruno ${ }^{1} \cdot$ Matteo Cesari ${ }^{2,3}$
}

Received: 6 January 2019 / Accepted: 27 February 2019

(c) Springer Nature Switzerland AG 2019

\begin{abstract}
In the present study, we explored the relationship between multimorbidity and frailty in a population of older individuals with cognitive disturbances attending a memory clinic. All subjects consecutively attending the Memory Clinic of the Department of Human Neuroscience, "Sapienza" University of Rome, between January 2017 and April 2018 for a first neurological evaluation were considered for the present analysis. Multimorbidity was defined as the simultaneous presence of two or more diseases in the same individual. A Frailty Index was computed by considering 44 age-related, multidimensional health deficits. Overall, 185 subjects were recruited in the study. A condition of multimorbidity was detected in $87.6 \%$ of the sample, whereas only the $44.6 \%$ of the study population was considered as frail. A poor agreement was observed between multimorbidity and frailty. The present findings confirm that counting diseases or health deficits may provide discordant information concerning the risk profile of older subjects.
\end{abstract}

Keywords Frailty $\cdot$ Multimorbidity $\cdot$ Cognitive disorders $\cdot$ Aging $\cdot$ Deficit accumulation

\section{Introduction}

The adoption of models of care consenting to adequately capture the health status and the risk profile of the older person has become a pressing need in most medical disciplines, well beyond the geriatric boundaries. In particular, a broader and holistic viewpoint is required today for improving our clinical and research approach to cognitive disturbances. This may help at disentangling the intricate network of underlying pathophysiological mechanisms, sustaining the design and conduction of more calibrated interventions, and improving the allocation of healthcare resources [1].

Interestingly, the clinical and biological complexity of the aging individual is increasingly estimated by mathematically counting his/her negative health attributes. The count

Marco Canevelli

marco.canevelli@gmail.com

1 Department of Human Neuroscience, "Sapienza" University, Viale dell’Università 30, 00185 Rome, Italy

2 Fondazione IRCCS Ca' Granda Ospedale Maggiore Policlinico, Milan, Italy

3 Geriatric Unit, Department of Clinical Sciences and Community Health, University of Milan, Milan, Italy of chronic diseases defines the concept of multimorbidity, intended as the co-occurrence of two or more diseases in the same individual [2]. The count of age-related health deficits (not restricted to traditional nosological entities, but also including signs, symptoms, and disabilities) is instead at the basis of the deficit accumulation model (or Frailty Index) proposed by Rockwood and Mitnitski [3]. The multimorbidity and deficit accumulation models share some commonalities and are frequently adopted interchangeably. After all, they were designed and respond to the same purpose of measuring the person's complexity. Nevertheless, the two follow distinct constructs and potentially provide different information in the clinical assessment of the older subject. In other words, they may differently inform and sustain clinical and public health interventions $[2,4]$.

In the present study, we explored the relationship between multimorbidity and frailty in a population of older individuals with cognitive disturbances attending a memory clinic. 


\section{Materials and methods}

All subjects consecutively attending the Memory Clinic of the Department of Human Neuroscience, "Sapienza" University of Rome, between January 2017 and April 2018 for a first neurological evaluation were considered for the present analysis. Participants underwent a detailed medical history collection and neurological and physical examination. Their global cognitive performance was assessed by means of the Mini Mental State Examination (MMSE) [5]. The Activities of Daily Living (ADL) [6] scale and the Instrumental ADL (IADL) [7] scale were adopted to evaluate their functional independence. Patients and/or their caregivers provided written informed consent for allowing the utilization of the collected data for research purposes.

The presence of the following 18 common medical conditions was recorded: osteoporosis; arthritis; hypertension; diabetes; dyslipidemia; ischemic heart disease; chronic heart failure; cancer; cardiac arrhythmia; chronic obstructive pulmonary disease; renal failure; gastric diseases; intestinal diseases; liver diseases; thyroid diseases; stroke; transient ischemic attack; peripheral artery diseases. The presence of these diseases was ascertained on the basis of: (a) self-reports concerning previous diagnoses and/or (b) available medical documents and/or (c) available medical prescriptions. Multimorbidity was defined as the simultaneous presence of two or more diseases in the same individual.

A Frailty Index was computed following a standard procedure [8] by considering 44 age-related, multidimensional health deficits (i.e., the above mentioned 18 chronic diseases plus symptoms, signs, and disabilities). Each item was coded as 0 (absence of the deficit) or 1 (presence of the deficit). The Frailty Index was then calculated as the ratio between the number of deficits exhibited by the participant and the number of considered deficits (i.e., 44). Thus, the score potentially ranged between 0 and 1 . A cut-off of 0.25 was used to categorically define a frailty condition [9].

The Cohen's kappa coefficient of agreement was tested between frailty and multimorbidity. Secondary analyses were conducted standardizing the cut-point of the two dichotomous variables of interest to the common values of $25 \%$ (that is raising the definition of multimorbidity to 4 or more diseases so to capture the same number of positive results as the FI) and $11 \%$ (that is lowering the definition of frailty to 5 or more deficits so to capture the same number of positive results as multimorbidity).

\section{Results}

Overall, 185 subjects (women 60.2\%) attended our service for a first cognitive assessment in the considered time period. They had a mean age of 75.1 [standard deviation (SD) 8.3] years. They overall presented a mild impairment of cognitive performance in the presence of functional independence, as indicated by mean MMSE, ADL, and IADL scores of 23.3 (SD 6.3), 5.4 (SD 1.3), and 5.0 (SD 2.7), respectively. A condition of multimorbidity was detected in the $87.6 \%$ of the sample, with participating subjects exhibiting a mean of 3.8 (SD 2.1) chronic diseases (Fig. 1). The Frailty Index ranged between 0.05 and 0.57 (median 0.22) (Fig. 1). Based on the adopted cut-point (i.e., $\geq 0.25$ ), $44.6 \%$ of the study population was considered as frail. A poor agreement was observed
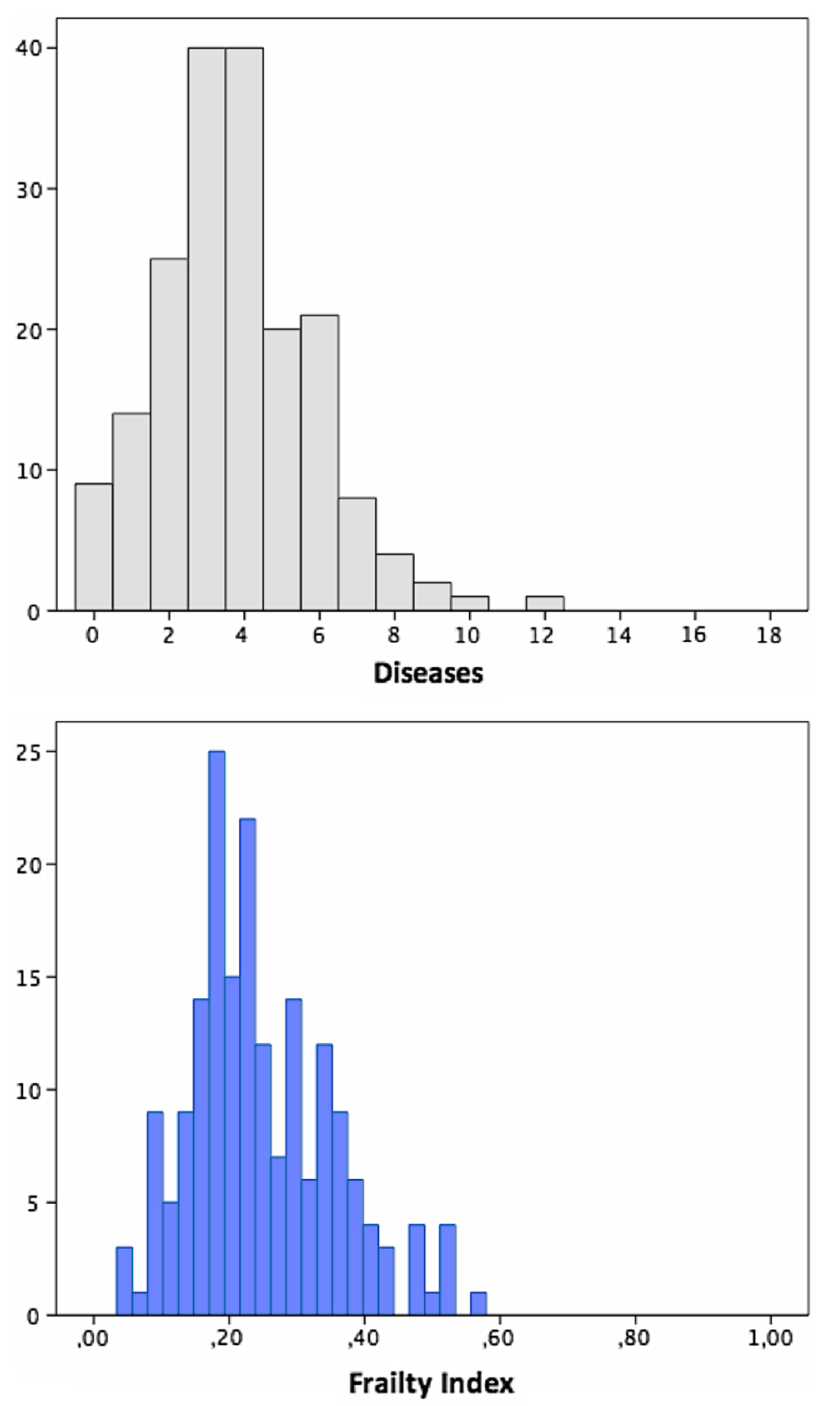

Fig. 1 Distributions of the individual number of diseases and Frailty Index scores in the study sample. Data are expressed as \% 
between multimorbidity and frailty $(k=0.08 ; p=0.06)$. In fact, only 93 subjects (50.3\%) simultaneously met the criteria for both or none of the two conditions, whereas 86 $(46.5 \%)$ individuals resulted as multimorbid but not as frail and $6(3.2 \%)$ resulted as frail but not as multimorbid.

Although markedly improved, the agreement between the two constructs remained relatively modest, both when the defining cut-point for frailty was lowered to $11 \%$ (i.e., $\mathrm{FI} \geq 0.11$, that is equivalent to the multimorbidity standard; $k=0.45 ; p<0.001)$ as well as when the defining cut-point for multimorbidity was increased to 4 or more diseases (i.e., $25 \%$, that is equivalent to the FI standard; $k=0.44$; $p<0.001)$.

\section{Discussion}

The present findings confirm that multimorbidity and frailty (here framed according to the deficit accumulation model) may provide different information concerning the clinical complexity of the older person and the stratification of his/her risk profile. In fact, despite being both (1) defined according to the arithmetical count of negative health attributes (i.e., diseases vs. deficits, respectively), and (2) similarly distributed in the study sample (Fig. 1), they exhibit a poor agreement in the identification of atrisk individuals. Consistently with the existing evidence, most frail individuals were also multimorbid whereas fewer multimorbid subjects presented a frailty condition [10]. This already observed discrepancy may be attributed to the existence of a temporal gap necessary for a condition of frailty to appear in multimorbid subjects. Such delta between the count of stand-alone diseases and the accumulation of deficits opens important research reflections. It may be considered as an indirect sign of resiliency at accumulating pathological changes [10]. It may also represent the result of largely unexplored interactions between diseases with a myriad of biological, psychological, social, and environmental determinants.

It might be argued that the difference between frailty and multimorbidity might be driven by the different rationale/ methodology applied in the definition of the critical thresholds of risk (i.e., $25 \%$ of deficits for frailty, and $11 \%$ of diseases for multimorbidity in our sample). However, the findings of the sensitivity analyses conducted standardizing the cut-point of the two variables to common values suggest that the two constructs should be considered as complementary rather than alternatives.

Of course, the lack of longitudinal data does not consent to appreciate which of the two models is better capable of mirroring the real health status of the individual and predicting his/her trajectories towards "hard" outcomes (e.g., mortality, hospitalization, institutionalization). Nevertheless, the observed ubiquitous presence of multimorbidity may somehow limit its discriminative role and overall clinical usefulness in our population. In other words, how can we draw clinical decisions (in terms of allocation of healthcare resources, frequency of follow-up, therapeutic choices) on the basis of a condition that is exhibited by nearly $90 \%$ of the subjects? Alternatively, a more rigid risk stratification can be obtained by adding to the model other subclinical and clinical deficits that, despite not meeting formal nosological frameworks, may strongly contribute to the health status and the clinical needs of the individual. In this regard, the Frailty Index allows the identification of a smaller number of subjects at increased risk for negative outcomes, potentially better supporting clinical decisions.

In conclusions, the present findings confirm how counting diseases or health deficits may provide discordant information concerning the risk profile of older subjects [4]. This should be carefully considered when planning and calibrating therapeutic and preventive interventions. The causal and temporal relationship between multimorbidity and deficit accumulation as well as their discriminating and predictive capacity should further be explored.

Funding None.

\section{Compliance with ethical standards}

Conflict of interest Marco Canevelli is supported by a research grant of the Italian Ministry of Health (GR-2016-02364975) for the project "Dementia in immigrants and ethnic minorities living in Italy: clinical-epidemiological aspects and public health perspectives" (ImmiDem). Matteo Cesari has received honoraria for presentations at scientific meetings and/or research funding from Nestlé and Pfizer. $\mathrm{He}$ is involved in the coordination of an Innovative Medicines Initiativefunded project [including partners from the European Federation Pharmaceutical Industries and Associates (Sanofi, Novartis, Servier, GSK, Lilly)]. The other authors have no conflict of interest to disclose.

Statement of human and animal rights All procedures performed in our study were in accordance with the ethical standards of the institutional research committee and with the 1964 Helsinki Declaration and its later amendments.

Informed consent Patients and caregivers (or legal guardians when necessary) provided their written informed consent for allowing the utilization of the collected data for research purposes (as required by the local Ethics Committee). Data used in the present analyses were exclusively retrieved from medical charts where information was recorded as part of the standard clinical routine.

\section{References}

1. Canevelli M, Cesari M, Remiddi F et al (2017) Promoting the assessment of frailty in the clinical approach to cognitive disorders. Front Aging Neurosci 9:36. https://doi.org/10.3389/fnagi .2017 .00036 
2. Vetrano DL, Calderón-Larrañaga A, Marengoni A et al (2018) An international perspective on chronic multimorbidity: approaching the elephant in the room. J Gerontol A Biol Sci Med Sci 73:13501356. https://doi.org/10.1093/gerona/glx178

3. Mitnitski AB, Mogilner AJ, Rockwood K (2001) Accumulation of deficits as a proxy measure of aging. Sci World J 1:323-336. https://doi.org/10.1100/tsw.2001.58

4. Cesari M, Pérez-Zepeda MU, Marzetti E (2017) Frailty and multimorbidity: different ways of thinking about geriatrics. J Am Med Dir Assoc 18:361-364. https://doi.org/10.1016/j.jamda .2016.12.086

5. Folstein MF, Folstein SE, McHugh PR (1975) "Mini-mental state". A practical method for grading the cognitive state of patients for the clinician. J Psychiatr Res 12:189-198

6. Katz S, Ford AB, Moskovitz RW et al (1963) Studies of illness in the aged. The index of ADL: a standardized measure of biological and psychological function. JAMA 185:914-919

7. Lawton MP, Brody EM (1969) Assessment of older people: selfmaintaining and instrumental activities of daily living. Gerontologist 9:179-186
8. Searle SD, Mitnitski A, Gahbauer EA et al (2008) A standard procedure for creating a frailty index. BMC Geriatr 8:24. https:// doi.org/10.1186/1471-2318-8-24

9. Kelaiditi E, Canevelli M, Andrieu S et al (2016) Frailty index and cognitive decline in Alzheimer's disease: data from the impact of cholinergic treatment use study. J Am Geriatr Soc 64:1165-1170. https://doi.org/10.1111/jgs.13956

10. Vetrano DL, Palmer K, Marengoni A et al (2018) Frailty and multimorbidity: a systematic review and meta-analysis. J Gerontol A Biol Sci Med Sci In Press. https://doi.org/10.1093/gerona/gly110

Publisher's Note Springer Nature remains neutral with regard to jurisdictional claims in published maps and institutional affiliations. 